\title{
Performance Evaluation of Anchor-based Range-based Localization Systems in Wireless Sensor Networks
}

\author{
Balaram Singh \\ Joint Venture College Computer \\ Education \\ B.B.Mahavidyalaya, Chandikhol \\ Utkal University, Odisha, India
}

\author{
Santosh Kumar Sahoo \\ Dept. of Comp. Sc. \& Engg. \\ Gurukul Institute of Technology, \\ BPUT,Odisha,India
}

\author{
Soumya Ranjan Pradhan \\ School of Comp. Engineering \\ KIIT University, Bhubaneswar \\ Odisha, India
}

\begin{abstract}
In the current scenario location estimation is a very challenging field in case of WSN, as the utility is very high in the WSN application with respect to various aspects. So, a simulation based survey is carried out in this paper using three mostly used range based techniques likely RSSI, TOA \& AOA, where the nodes are assumed to be static at the time of computation of their respective locations and some reference nodes are also considered for helping the location calculation, whose coordinates are known prior to the computation and is stored in the sensor nodes database.
\end{abstract}

\section{General Terms}

Wireless Sensor Networks, Location Estimation, Simulation, Survey.

\section{Keywords}

Location Estimation, Range Based, Anchor Based, RSSI, TOA, AOA

\section{INTRODUCTION}

Determining the location of a mobile device (cellular phone, user, sensor nodes/motes, and autonomous robots) is a fundamental problem in mobile computing. Being aware of the location of a device could either improve the performance of a communication network, or it could also be used to implement services relying on location information e.g. Location Based Services [1] etc. Wireless location services provide personalized services to mobile users based on their current location. The promise of new wireless location services heavily relies on accurate \& efficient location detection.

Localization [2] is the process by which an object determines its position with respect to spatial coordinates in a given field. It is also the process of finding the location of a mobile node in relation to its coverage/deployment area [3].It is about knowing the location of any network node at any time. Thereby, nodes can be either mobile, which means that their location can change, or static [10]. The process of finding location of the nodes is called localization [4][12].In this process we try to find out the approximate location of the sensor node with high accuracy. The process of localization collects information about the longitude, latitude and altitude of the sensor node.

We define the problem of localization as estimating the position or spatial coordinates of wireless sensor nodes. Localization is an inevitable challenge when dealing with wireless sensor nodes and a problem which has been studied for many years. Nodes can be equipped with a Global
Positioning System (GPS), but this is a costly solution in terms of volume, money and power consumption [1]

This paper is structured as follows. In section 2, Significance of the survey is given. The next section discusses about the basic approach of Location Estimation. In section 4 proposed works has been described in detail. In section 5 Performance evaluation \& simulation results are discussed. This paper concludes with Conclusions, Scope of this work \& Future Research Issue.

\section{SIGNIFICANCE OF THE SURVEY}

While much research has focused on developing different algorithms for localization, less attention has been paid to the problem of range measurement inaccuracy as well as performance of techniques in a particular environment. To the best of the authors' knowledge no such attempt has been made to compare some popular techniques with respect to a common environment.

In this paper, we discuss very often used techniques which are anchor based \& range based, set up in a common environment and try to find out the better solution for position estimation in the presence of range measurement inaccuracy for wireless sensor network.

\section{LOCATION ESTIMATION}

\subsection{Localization Techniques for WSN}

Localizations techniques for wireless sensor networks can be split into two families:

- Range-based techniques rely on nodes measuring distances or angles to reference nodes.

- Range-free techniques do not require nodes to measure distances or angles to reference nodes.

Range-based techniques rely directly on measured distances, and do not have specific topology requirements beyond basic geometric constraints (e.g., nodes must not be collinear (i.e., the angles between each of the nodes must be greater than $0 \circ)$ ). Conversely, range-free techniques do not require the nodes to measure distances or angles to neighbors, and instead rely only on connectivity as the basis for determining their position within the network. This reduces the hardware requirements of the nodes, and eliminates the possibility of measurement errors as no direct measurements are taken by the nodes

However, many of the range-free algorithms have certain topological requirements [11][13] for the network which must be fulfilled to ensure accurate localization estimates .Range free techniques can be further broken down into two groups. Area based localization [13][14] which works with areas to determine the most likely area a node is located in ,where as hop count based approaches count the number of hops to 
anchor nodes, which is then used to estimate distances to each of the anchor nodes, from which a position estimate can be calculated [15][16].

\subsection{Range Based Techniques}

In range based localization methods [4][12] depends upon distance or angle between nodes to obtain unknown nodes position. The 1st step is distance or angle measurement/ estimation is done. A number of approaches such as AOA, TOA, TDOA, and RSS exist. The $2^{\text {nd }}$ step is location estimation using Tri/Multi-lateration, Triangulation and MLE [8][9]. The advantage of this method is fine resolution but drawbacks are Extra hardware needed \& additional energy consumption.

These are following range based algorithms:-

\subsubsection{Angle of Arrival (AOA)}

An AOA [5][7] based positioning technique involves measuring angles of the node seen by reference nodes. In order to determine the location of a node in a 2D space, it is sufficient to measure the angles of the straight lines that connect the node and two reference nodes. AOA estimates the angle at which signals are received and uses simple geometric relationships to calculate node positions. Angles can potentially be estimated by using rotating directional beacons, or by using nodes equipped with phased array of RF or ultrasonic receivers [7]. Generally, AOA techniques provide more accurate localization result than RSSI based techniques but the cost of hardware of very high in AOA [7]. Also this method is affected by the multi-path effects, shadowing, scattering and also when there is no line of sight.

\subsubsection{Time of Arrival (TOA)}

Time-based positioning techniques rely on measurements of signal travel times between nodes. TOA [4][12] is a technique that allows locating a node by calculating the time of arrival of the signal from the node to more than one BS. The TOA can be estimated either by measuring the phase of the received narrowband carrier signal or by direct measurement of the arrival time of a wideband narrow pulse. The propagation time can be directly translated into distance, based on the known signal propagation speed [4]. These methods can be applied to many different signals, such as RF, Acoustic, Infrared and Ultrasound .If two nodes have a common clock, and the node receiving the signal can estimate the TOA of the incoming signal, which is time-stamped by the reference node. Therefore, this method requires the network to be synchronized (exact time synchronization)[12].

\subsubsection{Time Difference of Arrival (TDOA)}

The TDOA [4] technique can be employed when there is no synchronization between a given node and the reference nodes, but there is synchronization between reference nodes. The TDOA of two signals travelling between the given node and two reference nodes is estimated.This technique is conceptually quite simple. Basically 2 types of signals i.e. RF $\&$ Acoustic is used. It computes difference between arrival times to compute distance. Later Triangulation can be applied. This determines the location of the given node on a hyperbola with foci at the two reference nodes. A third reference node is needed to triangulate the position of the node. To achieve accurate positioning; the reference nodes must be synchronized in time. The drawbacks are Proper Calibration, expensive / energy intensive hardware [4].

\subsubsection{Received Signal Strength (RSS)}

RSS measures the power of the signal at the receiver and based on the known transmit power, the effective propagation loss can be calculated. Next by using theoretical and empirical models we can translate this loss into a distance estimate [2]. This method has been used mainly for RF signals. RSSI is a relatively cheap solution without any extra devices, as all sensor nodes are likely to have radios [4]. The performance, however, is not as good as other ranging techniques due to the multipath propagation of radio signals.

The RSSI model is based on the fact that signal strength diminishes with distance. Thus the distance between the source and the receiver could be found out by the strength of the radio signal received [2].RSS systems are very interesting because these are already in use in cellular and WLAN networks and can be used for urban and indoor geo location systems, without much modification to the existing one. But, a direct measurement of the distance from the RSS is not reliable, as the value of the RSS mainly depends on the pathloss model [17]. For WSN it should be combined with some position estimation techniques to give better approximation. Besides, an RSS measurement also depends on the channel characteristics. Therefore, RSS-based positioning algorithms are sensitive to channel parameters estimation [12].

\subsubsection{RSS profiling/Fingerprints}

Radio Fingerprinting, also known as Signature Database or Pattern Matching or Database Correlation [2], is another approach to localize an unknown node. The idea is that first determine the RSS (receive signal strength) values in several points and save these values in database and later use these value for comparison with received RSS values from an anchor node and thus estimating its location.

A wireless signal normally bounces off a variety of solid objects on the way to its destination, causing multipath interference. The same signal is received multiple times due to the delay caused by bouncing off objects and taking different paths to the destination Multipath fingerprinting takes advantage of this characteristic to describe signals that are received from certain locations. To employ this system, an operator must send test signals around to various locations so the nodes can record the fingerprints and create a database for comparison later on [2]. When there is new construction occurs in an area, the fingerprint will change and must be rerecorded.

In this technique, in addition to there being anchor nodes and non-anchor nodes, a large number of sample points are distributed throughout the coverage area of the sensor network [12]. At each sample point, a vector of signal strengths is obtained, with the $j$ th entry corresponding to the $j t h$ anchor's transmitted signal. Many entries of the signal strength vector may be zero or very small, corresponding to anchor nodes at larger distances (relative to the transmission range or sensing radius) from the sample point. The collection of all these vectors provides a map of the whole region. The collection constitutes the RSS model, and it is unique with respect to the anchor locations and the environment. The model is stored in a central location. By referring to the RSS model, a nonanchor node can estimate its location using the RSS measurements from anchors.

\subsection{Range Free Techniques}

Range free based techniques uses the information of topology $\&$ connectivity for location estimation. But these algorithms provide certain advantages i.e. low cost, small communication traffic, no extra hardware, \& flexible localization precision i.e. sometimes give more accuracy than range based methods which makes it appealing. 
These are following range free based algorithms:

\subsubsection{Binary Proximity}

It is one of the most basic location techniques, binary proximity involving a simple decision of whether two nodes are within reception range of each other or not [10]. A set of reference nodes are placed in the environment in some nonoverlapping manner. Either the reference nodes periodically emit beacons, or the unknown node transmits a beacon when it needs to be localized. If reference nodes emit beacons, these include their location IDs. The unknown node must then determine which node it is closest to, and this provides coarse grained localization. This Technique can be of considerable use in practice.

\subsubsection{Centroid}

N. Bulusu and J. Heidemann [10][11] first proposed a typical ,range-free, proximity-based, coarse grained localization algorithm, which makes full use of the connectivity of network to locate, uses anchor beacons, containing location information $\left(\mathrm{X}_{\mathrm{i}}, \mathrm{Y}_{\mathrm{i}}\right)$, to estimate node position. After receiving these beacons, a node estimates its location using Centroid formula. The proximity information can be used to greater advantage when the density of reference nodes is sufficiently high that there are several reference nodes within the range of the unknown node. The distinguished advantage of this Centroid localization scheme is its simplicity, ease of implementation, little power consumption. But its localization error has been prone to influence by the issues such as laying uniformity of anchor node and density of anchor nodes.

\subsubsection{Approximate Point in Triangulation (APIT)} Approximate Point in Triangulation (APIT) [15] is a range free approach of localization using geometric constraints. It requires a heterogeneous network of sensing devices where a small percentage of these devices (percentages vary depending on network and node density) are equipped with high powered transmitters and location information obtained via GPS or some other mechanism. These location-equipped devices are known as Anchors. Using beacons from these anchors, APIT employs a novel area-based approach to perform location estimation by isolating the environment into triangular regions between beaconing nodes A node's presence inside or outside of these triangular regions allows a node to narrow down the area in which it can potentially reside. By utilizing combinations of anchor positions, the diameter of the estimated area in which a node resides can be reduced, thus provides a good location estimate.

\subsubsection{Distance Vector Hop (DV-HOP)}

DV-HOP is first proposed by D.Niculescu and B.Nath [3][15].Anchor nodes generate packets including their position information and a flag which is initialized as 1 to figure the number of hops away from them. These packets are flooded in WSN. When they are transmitted by the relay nodes, the hop no. is increased by 1 . In this way, any node can determine the hop number from it to a certain anchor node. Similarly the anchor nodes can compute their hops to other anchors as well. The average distance per hop can be determined by a simple formula and then will be broadcast. When an unknown node receives it, the receiver will estimate its distance to the anchor node (average distance per hop*hop number). After it obtains three or more estimated values from anchor nodes, its location can be figured out.

In [16] the authors proposed an algorithm integrates RSSI and DV-hop. The new RDV-HOP algorithm makes the unknown node which is one hop distance from anchors calculate the distance from it to the neighbor anchors using RSSI method instead of DV-HOP method. Using the method, the estimate error in network is reduced. The limitation of the algorithm is that only neighbors of anchor can update.

\subsubsection{ROCRSSI}

Ring Overlapping based on Comparison of Received Signal Strength Indicator (ROCRSSI) [14] is a another range-free localization approach recently, aiming at dealing with the undetermined node problem of APIT and achieving higher estimation accuracy with lower communication over head. To calculate a position for sensor node $\mathrm{X}$, the ROCRSSI algorithm generates a set of rings each containing $\mathrm{X}$, computes the intersection of the rings, and uses the center of gravity of the intersection as the estimated position of $\mathrm{X}$. The motivation of ROCRSSI is to get more accurate estimation and reduce the number of .undetermined nodes, with small number of anchors. Since ROCRSSI does not try to map the received signal strength to absolute point-to-point distance, it belongs to range-free localization approaches. ROCRSSI only compares the relative strength of RSSI and does not depend on absolute RSSI values.

\section{PROPOSED WORK}

In this paper we primarily focused upon the range based, anchor based techniques in 2-D terrain. First we choose the range based techniques because of the better accuracy than of range free techniques. Anchor based technique is also simpler $\&$ effective than of Anchor free approach.

We first consider RSS because it is relatively simple, easy to implement \& cost effective as the sensor nodes inherently comes with built in transceivers which uses RF, so no added hardware cost i.e. this approach requires no additional hardware, and is unlikely to significantly impact local power consumption, sensor size and thus cost. It stands out as a fit medium for measuring node distances in WSNs.

Next we choose TOA and finally choose AOA. For position computation we use Trilateration/Triangulation.

\subsection{Basic Assumptions}

- The system consists of sensor nodes that are deployed uniformly \& randomly \& are fixed after deployment is in static environment which is a 2-D terrain.

- The terrain consist of the following type of sensor nodes i.e.

- Anchor Nodes (AN)-Beacon/Reference Nodes whose position is known $\&$ fixed

- Blind Nodes (BN)-Unknown Nodes which are to be localized.

- Sink Node (Sink)-It can be one of the anchor node which sends info back to the user.

- The Anchor nodes as well as Blind nodes are located within the maximum transmission range of the Sink node i.e. $\mathrm{R}_{\max }$.

- Each anchor node knows its position i.e. $\mathrm{A}\left(\mathrm{A}=\left(\mathrm{x}_{1}, \mathrm{y}_{1}\right)\right)$

- The nodes knows the local position information i.e. at least one hop neighbor.

- The anchors broadcast their location references periodically \& transmit at a fixed power level.

- The transmission range of the BNs is $r$ and the anchors is $R(R>=r>0)$, while the reception range of the Sink is $>=R$ is Rmax. 
- The communication links are symmetric i.e. if A can listen B then B also listen to A.

- All devices have Omni directional antennas.

- The line of sight (LOS) communication should exist between the transmitter and receiver.

- The receiver should able to receive RF as well as acoustic signals.

- The beacon is fitted with rotating directional antennae.

- All the angle related variables are in the range of $[0,2 \pi)]$.

- The transmissions between nodes are bounded by a maximum transmission range $d_{\max }$. Any packets received from a node outside of the transmission range are considered too weak to contribute.

- There are no major obstructions between the transmissions of any two nodes. Therefore, the AOA measurements can be assumed to have certain distribution centered on the direction of the line-ofsight (LOS).

\subsection{Procedure of Location Estimation}

\subsubsection{Received Signal Strength (RSS)}

- Let $\mathrm{A}(\mathrm{x} 1, \mathrm{y} 1)$ and $\mathrm{B}(\mathrm{x} 2, \mathrm{y} 2)$ be the 2 anchor nodes. $\mathrm{U}$ $(\mathrm{x}, \mathrm{y})$ be the unknown node/blind node whose position is to be calculated. Using Friis Transmission equation [17] we can calculate free space path loss value in $d B$ or received signal strength RSS in $d B$ [RSSI in $\mathrm{dBm}$ ] if frequency $\mathrm{f} \&$ distance $\mathrm{d}$ is known.

$$
\begin{aligned}
& P L_{F S}(\mathrm{~dB})=--27.55 \mathrm{~dB}+20 * \log _{10}(\mathrm{f})+20 * \log _{10}(\mathrm{~d}) \\
& \text { Where } \mathrm{f}=\text { frequency in } \mathrm{MHz} \\
& \mathrm{d}=\text { distance in Meters }
\end{aligned}
$$

- We consider the path loss values i.e. received signal strength (RSS) from the blind node to anchor nodes are known to us \& according to literature used in simulation. Using the path loss value we convert it to distance using the following formula:

$$
\begin{aligned}
& d_{1}=10^{\left(\frac{P L-P L d 0-20 * \log _{10}(f)}{20}\right)} \\
& d_{2}=10^{\left(\frac{P L-P L d 0-20 * \log _{10}(f)}{20}\right)}
\end{aligned}
$$

- Here $\mathrm{d} 1$ is the distance between unknown node $\mathrm{U}(\mathrm{x}$, y) and anchor node A (x1, y1) \& d2 is the distance between Unknown node $\mathrm{U}(\mathrm{x}, \mathrm{y})$ and anchor node $\mathrm{B}(\mathrm{x} 2, \mathrm{y} 2)$. Since A \& B 's positions are known so by using 2 point distance formula we can calculate the value of $\mathrm{x} \& \mathrm{y}$. Putting the value of $\mathrm{d} 1 \& \mathrm{~d} 2$ we can get 2 equations as

$$
\begin{aligned}
& d 1^{2}=(x-x 1)^{2}+(y-y 1)^{2} \\
& d 2^{2}=(x-x 2)^{2}+(y-y 2)^{2}
\end{aligned}
$$

- On solving above equations we can get the values of $\mathrm{U}(\mathrm{x}, \mathrm{y})$ and $\mathrm{U}\left(\mathrm{x}^{\prime}, \mathrm{y}^{\prime}\right)$, then we calculate rms. error to find the error difference i.e. between actual position vs. estimated position in this technique. The error can be calculated as:

$$
\operatorname{error}_{r s s}=\sqrt{\left(x-x^{\prime}\right)^{2}+\left(y-y^{\prime}\right)^{2}}
$$

\subsubsection{Time of Arrival (TOA)}

- Let $\mathrm{A}(\mathrm{x} 1, \mathrm{y} 1)$ and $\mathrm{B}(\mathrm{x} 2, \mathrm{y} 2)$ be the 2 anchor nodes. $\mathrm{U}$ $(\mathrm{x}, \mathrm{y})$ be the unknown node/blind node whose position is to be calculated.

- Here we used one way propagation of time of arrival. The time of arrival can be calculated as

$$
\operatorname{toa}_{i}=\left(t_{i}-t\right)
$$

Where: $i=1,2,3, \ldots \ldots n$,

$\mathrm{t}=$ signal departing time from the unknown node $\mathrm{U}$

$t_{i}=$ signal arriving time at the anchor node AN

- According to literature, if TOA is known from transmitter to receiver \& velocity of transmission is also known then we can calculate the distance between them using the following formula i.e.

$$
d_{i}=c * \operatorname{toa}_{i}
$$

Where: $i=1,2,3, \ldots . . n$,

$$
\mathrm{c}=3 * 10^{8} \mathrm{~ms}^{-1}=\text { signal propagation speed; }
$$

- After we got 2 TOA i.e. TOA1 and TOA2 it is put in to following equation and we calculate 2 distances as $d_{1} \& d_{2}$

$$
\begin{aligned}
& d_{1}=c * t o a_{1} \\
& d_{2}=c * t^{o o} a_{2}
\end{aligned}
$$

- On solving above equations we can get the value of $\mathrm{U}(\mathrm{x}, \mathrm{y})$.Similarly we can also get the value of $\mathrm{U}\left(\mathrm{x}^{\prime}\right.$, $\left.y^{\prime}\right)$, then we calculate rms. error to find the error difference i.e. between actual position vs. estimated position in this technique. The error can be calculated as:.

$$
\operatorname{error}_{T O A}=\sqrt{\left(x-x^{\prime}\right)^{2}+\left(y-y^{\prime}\right)^{2}}
$$

\subsubsection{Angle of Arrival (AOA)}

- Let $\mathrm{A}(\mathrm{x} 1, \mathrm{y} 1)$ and $\mathrm{B}(\mathrm{x} 2, \mathrm{y} 2)$ be the 2 anchor nodes. U $(\mathrm{x}, \mathrm{y})$ be the unknown node/blind node whose position is to be calculated.

- The AOA can be calculated as

$$
\begin{aligned}
& \theta_{1}=\tan ^{-1}\left(\frac{y-y 1}{x-x 1}\right) \\
& \theta_{2}=\tan ^{-1}\left(\frac{y-y 2}{x-x 2}\right)
\end{aligned}
$$

- From the above equations we have

$$
\begin{aligned}
& \tan \theta_{1}=\left(\frac{y-y 1}{x-x 1}\right) \\
& \tan \theta_{2}=\left(\frac{y-y 2}{x-x 2}\right)
\end{aligned}
$$

- On solving the eqns. we can get the value of $U\left(x^{\prime}, y^{\prime}\right)$.

- Now we have the values of $U(x, y)$ and $U\left(x^{\prime}, y^{\prime}\right)$, then we calculate rms. error to find the error difference i.e. between actual position vs. estimated position in this technique. The error can be calculated as :

$$
\operatorname{error}_{A O A}=\sqrt{\left(x-x^{\prime}\right)^{2}+\left(y-y^{\prime}\right)^{2}}
$$




\section{SIMULATION \& RESULTS}

Simulation was carried out using MATLAB $^{\circledR}$ 7.9.For Simulation we take 2 anchor nodes instead of 3 anchor nodes as generally chosen. We know the anchor node positions by manual placement. The anchor nodes as well blind node is positioned within a macro cell having maximum range of 2000 meters.

We compare the RSSI, TOA \& AOA techniques in terms of its root mean square error. The final outcome of the simulation is enlisted in the following table i.e.

Table 1. Error range VS Percentage of Errors IN AOA, TOA \& RSSI

\begin{tabular}{|c|c|c|c|}
\hline Error Range & \multicolumn{3}{|c|}{ Percentage (\%) Of Errors in } \\
\cline { 2 - 4 } in Meters & $\boldsymbol{A O A}$ & $\boldsymbol{T O A}$ & $\boldsymbol{R S S I}$ \\
\hline $0-50$ & 50 & 50 & 100 \\
\hline $51-100$ & 50 & 40 & 0 \\
\hline $101-200$ & 0 & 10 & 0 \\
\hline $201-500$ & 0 & 0 & 0 \\
\hline
\end{tabular}

Here from Table I we can analyze that the no of rms. errors in percentage is given vs. range of errors in meters. \& there are 4 nos. of error ranges i.e. $<50$ (less than 50 meter), 50-100 meter, 100-200 meter \& 200-500 meter.

From the simulation we found that if distance $<50$ meter, then the errors in AOA are approx.50\%, the errors in TOA are approx. $50 \%$ \& the errors in RSSI are almost $100 \%$, so most of the RSSI errors are within this range, again if the distance is between 50-100 meter we found that the approx $50 \%$ of AOA errors as well as approx.40\% of TOA errors exists \& no RSS errors are within this range. We can see that if the error range is of 100-200 meter only $10 \%$ of TOA errors exist \& no other error exists. In the range of 200-500 meter none of the technique shows error.

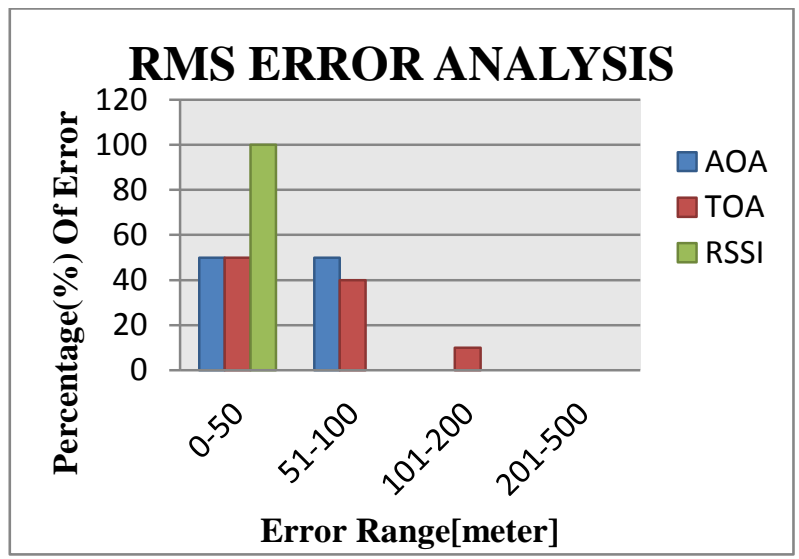

Fig 1: Error range vs. Percentage of Errors in AOA, TOA \& RSSI (RMS Error Analysis)

From the figure-1, we see that most of the RSSI errors are within the distance of 50 meters so RSSI is the best technique suited for wireless sensor nodes because the errors within 50 meter are not considered as error, then comes the AOA and TOA is more error prone.

\section{ACKNOWLEDGMENTS}

We acknowledge all the authors who helped us in this work directly/indirectly by their publications. Also we here thank all the sites which gave us great support in the form of materials.

\section{CONCLUSION}

Though every procedure got their own advantages and limitations, RSSI technique is proved to be the least erroneous technique in the above simulation and hence the accuracy is shown to be very high in case of WSN but still high computation is required for the calculation of the location just because it requires a huge number of parameter in the process, which leads to the higher consumption of power. But in TOA technique though the error rate is high but this is the easiest way to calculate the location so far and hence less power consuming, where as the AOA technique though shows the better accuracy than the TOA, the angle calculation actually is a very tedious process including the hardware cost as well for angle calculations.

\section{SCOPE OF THE WORK}

The advantage of this work is no need of using three Anchor nodes for participation in estimating the location of unknown node as used in conventional methods. This work reduces the cost of deploying an extra Anchor node where as the limitation is since it depends upon the LOS communication between the Unknown node and Anchor nodes which is not feasible always.

\section{FUTURE WORKS}

At the time of simulation the Unknown node as well as the Anchor nodes are assumed to be present within a macro cell until location of unknown node is estimated but in reality the Unknown node may be mobile, so error estimation may varies must be able to support it. Future work may include error analysis in case of both Anchor node \& Unknown node are mobile in nature.

\section{REFERENCES}

[1] Paul J. Kuhn ," Location Based Services in Mobile Communication Infrastructures ", Electronic Communication (AEU) 58, 2004.

[2] A. Roxin, J. Gaber, M. Wack, A. Nait SidiMoh ," Survey of Wireless Geolocation Techniques ", IEEE Globecom Workshop, :1-9, 2010.

[3] Niculescu, D, and Nath, B ," Ad Hoc Positioning System (APS) Using AOA ", 2003

[4] Zhetao Li, Renfa li et al ," Survey of Localization Techniques in Wireless Sensor Network ", Information Technology Journal 9(8):1754-1757,2010

[5] Minghui Li, Kwok Shun Ho, Gordon Hayward , " Accurate Angle-of-Arrival Measurement Using Particle Swarm Optimization ", Wireless Sensor Network, 2: 358$364,, 2010$

[6] J. Jasper Gnana Chandran \& S. P. Victor ," An Energy Efficient Localization Technique Using Particle Swarm Optimization in Mobile Wireless Sensor Networks ". American Journal of Scientific Research, ISSN 1450223X Issue 8: 33-48, 2010

[7] Kułakowski P et al ," Angle-of-arrival localization based on antenna arrays for wireless sensor networks ", Elsevier, Computers \& Electrical Engineering (2010). 
[8] Raghavendra V. Kulkarni, \& Ganesh Kumar Venayagamoorthy ,"Bio-inspired Algorithms for Autonomous Deployment and Localization of Sensor Nodes ",IEEE Transactions On Systems, Man, And Cybernetics-Part C: Applications And Reviews, Issue 5, Volume 40, 2010.

[9] Aysegul Alaybeyoglu et al ,"Tracking Fast Moving Targets in Wireless Sensor Networks ", IETE Technical Review : 46-53,Vol 27, Issue 1, Jan-Feb 2010

[10] N. Bulusu, J. Heidemann and D. Estrin ,"GPS-Less Low Cost Outdoor Localization for Very Small Devices ". IEEE Personal Communications Magazine, : 28-34, Vol. 7, No. 5, 2000, .

[11] Jijun Zhao, Hua Li, Zhiyuan Tang, Xiang Sun ," Research on WSN Double-Radius Localization Algorithm Based on Partition Judgment Mechanism ", Wireless Sensor Network, 2,: 639-644,2010.

[12] Isaac Amundson and Xenofon D. Koutsoukos ," A Survey on Localization for Mobile Wireless Sensor Networks ", MELT 2009, LNCS 5801 : 235-254, 2009
[13] J.M.Sánchez-Matamoros, J.R.Martínez-de Dios and A. Ollero, "Cooperative localization and tracking with a camera based WSN ". IEEE International Conference on Mechatronics, April 2009.

[14] Chong Liu, Tereus Scott, Kui Wu, Dan Hoffman," Range-Free Sensor Localization with Ring Overlapping Based on Comparison of Received Signal Strength Indicator".2007

[15] Tian He, Chengdu Huang, Brian M. Blum, John A. Stankovic, Tarek Abdelzaher "Range-Free Localization Schemes for Large Scale Sensor Networks" , ACM MobiCom '03, September 14-19, 2003

[16] Shuang Tian, Xinming Zhang, Pengxi Liu, Peng Sun, Xinguo Wang," A RSSI based DV-hop Algorithm for Wireless Sensor Networks", IEEE, 2007.

[17] T. S. Rappaport, Wireless Communications: Principles and Practice, 2nd ed. Prentice Hall PTR, 2009

[18] Xiangyang Li,Wireless Ad Hoc Sensor Networks:Theory and Applications,Cambridge University Press,2008 